\title{
A REVIEW OF 72 CONSECUTIVE CASES OF ACANTHAMOEBA KERATITIS, 1984-1992
}

\author{
A. S. BACON, D. G. FRAZER, J. K. G. DART, M. MATHESON, L. A. FICKER and P. WRIGHT \\ London
}

\begin{abstract}
SUMMARY
A review of consecutive cases of Acanthamoeba keratitis presenting since 1984 was undertaken in order to assess prognostic factors, the success of culture procedures and the outcome of medical and surgical management, with reference to current clinical practice. Seventy-two consecutive cases (77 eyes) of Acanthamoeba keratitis have been managed. Sixty-four patients were contact lens wearers, 28 of these wearing disposable lenses. Superficial corneal involvement and perineural infiltrates were common in those diagnosed less than a month after first symptoms, designated 'early' presentation. Ring infiltrates and ulceration with stromal lysis characterised those presenting at 1-2 months ('intermediate') or after 2 months ('late'); these groups also progressed more frequently to hypopyon, scleritis, glaucoma and cataract formation. Positive corneal cultures were obtained in 10 of $14(71 \%)$ intermediate and 17 of $23(74 \%)$ late cases; early cases underwent epithelial biopsy but formal trephine biopsy was not usually justified (1 of 35 cases) and only 19 of $35(54 \%)$ were tissue-positive. Microbial coisolates were obtained from 20 corneas. Thirty-four penetrating keratoplasties were performed in 23 eyes, 21 whilst inflamed and 13 when quiet. Of 13 failures in inflamed eyes, 9 were due to recurrence of Acanthamoeba infection. Medical cure is known to have been achieved in 64 of $73(88 \%)$ eyes, 4 of the original 77 having been lost to follow-up abroad. Fifty-eight of 73 eyes (79\%) achieved a final visual acuity of $6 / 12$, and of the culture-positive cases, 32 of $46(70 \%)$ achieved 6/12. Since 1984 an effective biguanide-propamidine treatment combination has been introduced, cases have been diagnosed increasingly early and consequently the time taken for medical cure has decreased. The prognosis of this potentially blinding keratitis has improved due to heightened clinical suspicion resulting in early diagnosis, a rigorous scheme of investigation and proven management strategies.
\end{abstract}

Acanthaemoeba infection of the cornea is known to result in a wide spectrum of corneal disease requiring different management strategies. Prior to effective anti-amoebal

Correspondence to: Annette Bacon, FRCOphth, Moorfields Eye Hospital, City Road, London EC1V 2PD, UK. therapy and early diagnosis, the organism was associated with devastating ocular damage, which still occurs in a small number of patients, influenced by various factors such as misdiagnosis, ${ }^{1-5}$ inappropriate antimicrobial treatment, excessive topical steroid prior to correct diagnosis ${ }^{6,7}$ and resistant organisms. ${ }^{8,9}$ Acanthamoebae are good opportunists because of their ability to encyst, when they may become more resistant to the agents used..$^{10}$ If the infection proceeds without appropriate early treatment, acanthamoebae appear to invade progressively deeper into the corneal stroma and are presumed to feed on keratocytes. Other tissues may be invaded, and acanthamoebae have even been isolated from the anterior chamber. ${ }^{11}$ The success of topical agents may then be limited as their concentrations are reduced in these deeper target tissues; the importance of the role of currently available systemic anti-amoebal drugs is not yet clear.

The number of cases of Acanthamoeba keratitis presenting per year in the United Kingdom appears to be rising, possibly because of increasing awareness of the condition and direct referral to specialist centres. In addition there has been a gradual rise in the number of disposable lens wearers affected, 10 of 31 corneal culture-positive patients presenting between January 1989 and April 1992 wearing disposable lenses (A. S. Bacon et $a l$., unpublished data, presented at the Oxford Congress of Ophthalmology 1992). The need for education and improved lens care practices has been identified, particularly amongst the growing number of disposable lens wearers.

Ficker reported 11 cases of Acanthamoeba keratitis presenting at Moorfields Eye Hospital between 1986 and $1987 .{ }^{12}$ We recently confirmed her finding that early treatment using a combination of propamidine isethionate and neomycin is effective and associated with an excellent prognosis; ${ }^{6} 15$ consecutive patients diagnosed before 4 weeks from first symptoms regained 6/12 vision at least and only 1 with resistant disease required penetrating keratoplasty. The diagnosis of Acanthamoeba keratitis is high on the differential diagnosis list for atypical keratitis, especially in contact lens wearers, and therefore more patients are diagnosed early. 
We now present updated findings on 72 patients (77 eyes) seen at Moorfields Eye Hospital between March 1984 and September 1992, in order to chart some of the changes in the pattern of presentation and diagnosis over these years. The primary aims are to establish prognostic factors in relation to the clinical features, the results of laboratory investigations and the outcome of medical and surgical management.

\section{PATIENTS AND METHODS}

A database of all patients diagnosed as having Acanthamoeba keratitis was kept prospectively from January 1990. Details from the case records of patients who had presented between 1984 and 1989 were added retrospectively. At each outpatient visit clinical signs and treatment details were recorded.

The patients were divided into three groups on the basis of the interval between first symptoms and the introduction of anti-amoebal therapy, because the severity of disease and the final visual outcome appear related to this. ${ }^{6}$ The groups were: 'early', those correctly diagnosed and starting treatment within a month (28 days) of first symptoms; 'intermediate', those starting appropriate treatment between 1 and 2 months after symptoms; and 'late', those presenting or being diagnosed after 2 months. Analysis of the presenting signs, clinical diagnosis, laboratory findings, complications and outcome was performed.

\section{Diagnostic Criteria}

Forty-six of 72 patients $(64 \%)$ had culture-positive corneal tissue. A further 8 patients (11\%) had positive cultures of Acanthamoeba from the contact lens, case or solutions in association with keratitis of presumed amoebal aetiology. Of 18 further patients with presumed Acanthamoeba keratitis, 9 (13\%) presented with perineural infiltrates (PNI), regarded as positive confirmation of the diagnosis (see Discussion). The last 9 (13\%) were included on clinical grounds, having atypical keratitis in combination with typical symptoms and signs such as disproportionate pain $(n=9)$, lens-wear $(n=9)$, ring infiltrates $(n=3)$, dendriform ulceration $(n=3)$, limbitis $(n=9)$ and hypopyon $(n=2)$; they all exhibited a good clinical response to appropriate anti-amoebal therapy.

\section{Diagnostic Procedure}

A sheet of epithelium (epithelial biopsy) was removed from the affected cornea and divided in half. One specimen was placed in formol saline and sent for histopathological analysis; the other was sent to the microbiology department. Microscopy was performed using calcofluor white $^{13}$ and immunostains. ${ }^{14}$ The specimen was partly homogenised and plated onto a non-nutrient agar plate which was then flooded with live Escherichia coli and the plates were incubated at $37^{\circ} \mathrm{C}$ for 72 hours. Acanthamoebae were identified on binocular microscopy by the presence of trophozoites with vacuoles. If there was no growth at this stage the plates were incubated for a further 72 hours at $30^{\circ} \mathrm{C}$, then if necessary at room temperature, and were not finally discarded for 3 weeks. If there were stromal infiltrates, scrapes from the advancing edge (off the visual axis where possible) were also taken. The contact lens, lens case and all available solutions were also sent for examination and culture. As a microbial screen, scrapes and biopsies were also plated onto blood agar for aerobic and reduced-oxygen culture, nutrient, thioglycollate and Robertson's cooked meat broths, Sabouraud and Lowenstein-Jensen media.

Formal corneal biopsy was performed if scrapes were negative in cases where the clinical response to treatment was poor. Biopsy samples and corneal discs from penetrating keratoplasties were examined by microscopy with the special stains and cultures described above.

Cultures of Acanthamoeba from all sources were sent to Mr. Simon Kilvington (Public Health Laboratory, Bath, UK) for storage and in vitro drug sensitivity testing in cases with a poor response to therapy.

\section{Treatment}

Combination anti-amoebal treatment was used. Propamidine and neomycin were the first line treatment until recently (1990-1), when the combination of propamidine isethionate and polyhexamethylene biguanide (PHMB) ${ }^{15}$ became more commonly used. Intensive treatment was commenced, with drops given at up to half-hourly intervals and reduced as the clinical response began. When the eye was finally free of any clinical signs of inflammation or infection, one anti-amoebal (formerly propamidine but now often PHMB) was continued at a level of 2-4 times daily dose for 6-8 weeks. The regime was altered if there was a deterioration or no response, particularly if the in vitro tests confirmed resistance to the drugs used. Topical paromomycin $2.5 \%$, clotrimazole $1 \%$, miconazole $1 \%$ and oral itraconazole (100 mg once daily) and ketoconazole (200 mg once daily) were common agents added.

Oral flurbiprofen $50-100 \mathrm{mg}$ two or three times daily was given for the treatment of pain and inflammation including limbitis and scleritis. Topical steroids (prednisolone $0.3 \%$ or dexamethasone $0.1 \%$ ) were reserved for eyes with specific indications such as uveitis, indolent ulcers, stromal lysis, or after keratoplasty; systemic steroids were reserved almost exclusively for scleritis, in combination with flurbiprofen and topical steroids.

The indications for penetrating keratoplasty changed over the years 1984-1992 as medical treatment showed success. Although reduction of the infective load was originally considered important, the current indications for keratoplasty in an inflamed eye are tectonic only. In a quite eye, however, keratoplasty may be done for optical reasons.

\section{RESULTS}

\section{Source of Patients}

A diagnosis of Acanthamoeba keratitis was made in 72 patients (77 infected eyes) at Moorfields Eye Hospital between March 1984 and September 1992. Twenty-eight 


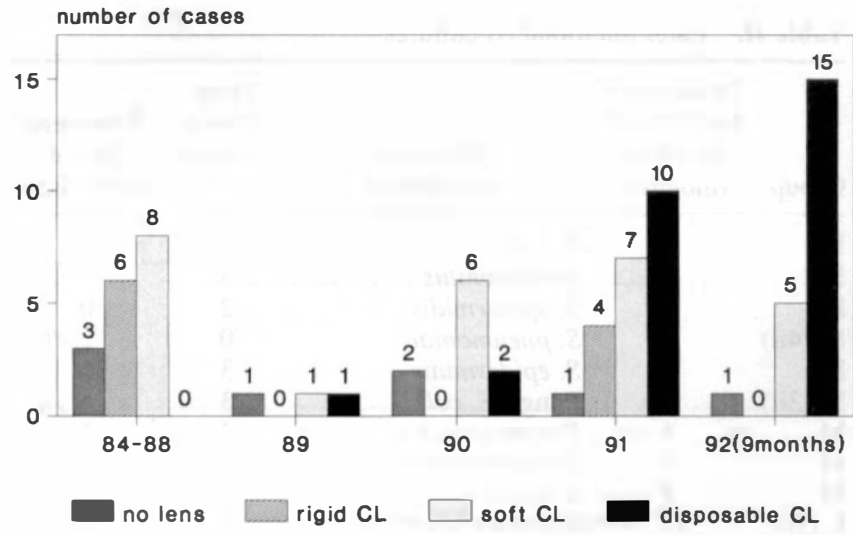

Fig. 1. Contact lens (CL) wear associated with cases of Acanthamoeba.

patients (39\%) were seen at Moorfields in the casualty department or the External Disease Service at their first presentation. Forty-four $(61 \%)$ were tertiary referrals from other centres: 12 from London and another 15 from the South East of England, 3 from the South West, 3 from the Midlands, 3 from the North East, 2 from the North West and 6 from abroad.

\section{Predisposing Factors}

Sixty-four patients $(89 \%)$ were contact lens wearers (Fig. 1). The ratio of soft to rigid contact lenses of 2.7:1 was actually lower than the $4: 1$ worn by the population attending Moorfields ${ }^{16}$ in a 1987-8 study. The number of patients who wore disposable lenses rose steadily from 1989 to 1992.

\section{Diagnostic Delay}

The interval between first symptoms and the diagnosis of Acanthamoeba keratitis (diagnostic interval) fell from 1985 (mean 180 days) to 1992 (mean 9.3 days) for those patients presenting directly to Moorfields (see Fig. 2). Overall there were 35 patients ( 36 eyes) in the early group, 14 ( 17 eyes) in the intermediate and 23 ( 24 eyes) in the late. In the late group, patients presented a mean 3.9 months after first symptoms (range 2-15 months).

A misdiagnosis of herpes simplex keratitis was made in 21 of $30(70 \%)$ patients between 1984 and 1990. In 1991

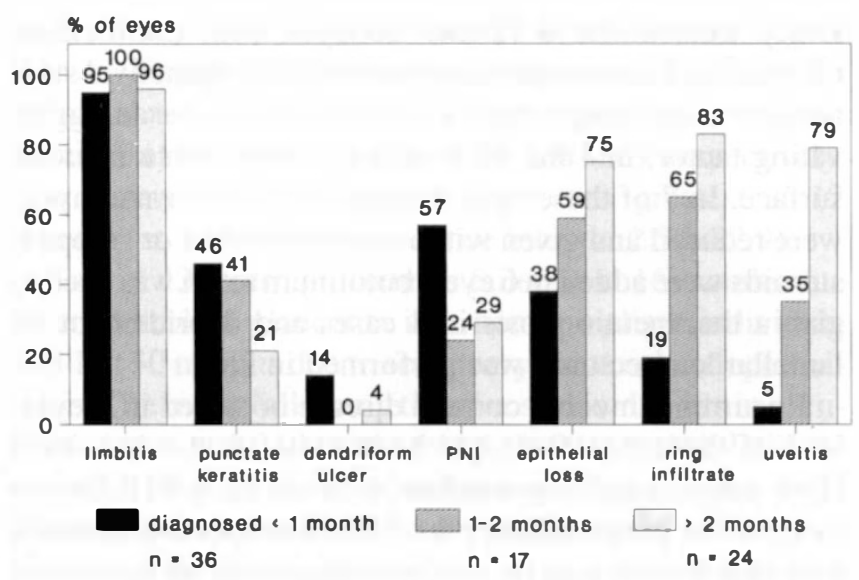

Fig. 3. Presenting features of Acanthamoeba keratitis.

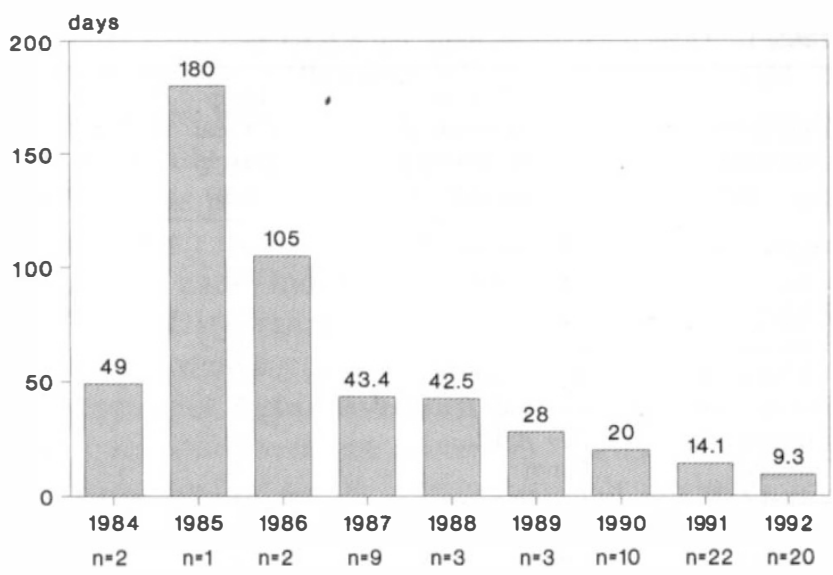

Fig. 2. Diagnostic interval (days from onset to treatment) for primary referrals.

there was a similar proportion $(16 / 21,76 \%)$, although Acanthamoeba keratitis was the primary diagnosis in 3 cases (14\%). In 1992, however, only 2 of 19 (11\%) cases were misdiagnosed as herpes simplex keratitis and 9 of 19 (47\%) were correctly diagnosed as Acanthamoeba keratitis.

\section{Clinical Features}

The presenting signs were markedly different in the early, intermediate and late groups (Fig. 3), except that limbitis was almost universally present. Early disease was characterised by limbitis, perineural infiltrates and superficial epithelial changes, with few ring infiltrates and little anterior uveitis. Patients in the later groups, however, demonstrated frank ulceration, ring infiltrates and anterior uveitis, often with hypopyon; a small number of patients demonstrated endothelial plaques causing corneal oedema. Perineural infiltrates were less common than in early disease.

Further evolution of severe features of the disease, including ring ulcers, abscesses, hypopyon, scleritis, glaucoma, cataracts and secondary microbial infection, occurred more commonly in later-presenting disease (Fig. 4).

\section{Rate of Culture-Positivity}

A positive tissue diagnosis of Acanthamoeba was made in

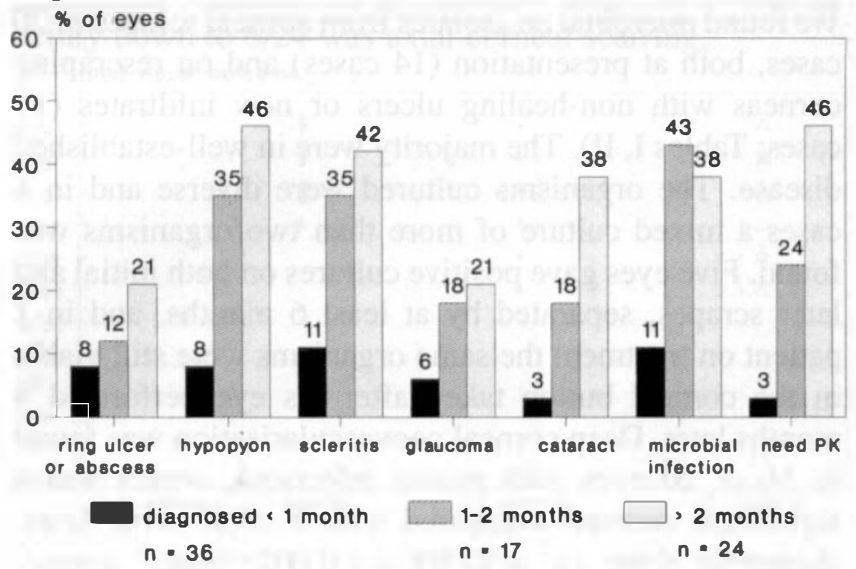

Fig. 4. Late features of Acanthamoeba keratitis. 
Table I. Other microbes on initial corneal scrape

\begin{tabular}{|c|c|c|c|}
\hline $\begin{array}{l}\text { Time from } \\
\text { symptoms } \\
\text { to scrape }\end{array}$ & $\begin{array}{c}\text { Microbes } \\
\text { co-cultured } \\
\text { (microscopy } \\
\text { in parentheses) }\end{array}$ & $\begin{array}{l}\text { Deep } \\
\text { corneal } \\
\text { vessels } \\
(\mathrm{mm})\end{array}$ & $\begin{array}{l}\text { Treatment } \\
\text { (no. of } \\
\text { antibiotics) }\end{array}$ \\
\hline 3 days & $(\mathrm{G}+$ cocci $)$ & 0 & 0 \\
\hline 5 days & $(\mathrm{G}-\operatorname{rod})^{\mathrm{a}}$ & 4 & 2 \\
\hline 7 days & $(\mathrm{G}+$ cocci $)$ & 0 & 0 \\
\hline 3 weeks & E. coli & 0 & 0 \\
\hline 4 weeks (4i) & $(G-\operatorname{rod})^{a}$ & 0 & 2 \\
\hline 5 weeks (1R) & $\left(\mathrm{G}-\right.$ rod) Bacillus ${ }^{\mathrm{a}}$ & 5 & 2 \\
\hline 5 weeks (1L) & $(G-\operatorname{rod})^{\mathrm{a}}$ & 3 & 2 \\
\hline 5 weeks & Fungi & 4 & 3 \\
\hline 6 weeks (2L) & Pseudomonas & 2 & 2 \\
\hline 2 months (2R) & Pseudomonas ${ }^{\mathrm{a}}$ & 2 & 2 \\
\hline 2 months ( $3 i)$ & Fungi, E. coli ${ }^{\mathrm{a}}$ & 3 & 4 \\
\hline 3 months & S. epidermis, Fungi ${ }^{\mathrm{a}}$ & 1 & 4 \\
\hline 4 months & $S$ aureus, $C$. xerosis ${ }^{\mathrm{a}}$ & 2 & 0 \\
\hline 8 months & (G- rod), S. epidermidis & 3 & 2 \\
\hline
\end{tabular}

$(1 \mathrm{R} / 1 \mathrm{~L})$ etc. indicates same patient, right/left eyes; (3i/3ii) etc. indicates same eye on different occasions; G+, G- indicates Gram-positive and Gram-negative respectively.

${ }^{a}$ Frank abscess.

a total of 46 of 72 cases (64\%). Laboratory identification of the organism was more successful in those with longerstanding disease: 17 of 23 (74\%) of those presenting late, 10 of $14(71 \%)$ of the intermediate group and 19 of 35 early cases (54\%) were culture-positive; repeat epithelial and stromal scrapes and formal trephine biopsies were, however, performed more commonly in late and intermediate cases than in early, where an improving clinical picture rarely justified further invasive investigation. Cultures from the second eye in bilateral cases were not usually pursued. Cultures were positive from the lens or care systems in a further $8(11 \%)$ cases.

The success of laboratory investigations per se was as follows: 33 of 66 initial epithelial biopsies/scrapes from subjacent infiltrates were culture positive (32/66 microscopy-positive), 11 of 20 formal biopsies were culturepositive (13/20 on microscopy) and 9 of 15 host cornea buttons were culture-positive (10/15 on microscopy). Of samples from lens cases and solutions, 32 of 41 yielded cultures of Acanthamoeba (29/41 positive on microscopy).

\section{Microbial Co-isolates}

We found microbial co-isolates from corneal scrapes in 20 cases, both at presentation (14 cases) and on rescraping corneas with non-healing ulcers or new infiltrates (11 cases; Tables I, II). The majority were in well-established disease. The organisms cultured were diverse and in 8 cases a mixed culture of more than two organisms was found. Five eyes gave positive cultures on both initial and later scrapes, separated by at least 6 months, and in 1 patient on treatment the same organisms were still viable in the corneal button taken after his eye perforated 4 months later. Deep corneal neovascularisation was found in 16 of 20 eyes with mixed infections, which was a significant increase compared with 8 of 57 with Acanthamoeba alone $\left(\chi^{2}=27.03, p<0.001\right.$; Yates' correction). The morbidity appeared prolonged in those with
Table II. Later microbial co-cultures

\begin{tabular}{|c|c|c|c|c|}
\hline Group & $\begin{array}{c}\text { Duration of } \\
\text { keratitis till } \\
\text { reculture } \\
\text { (months) }\end{array}$ & $\begin{array}{l}\text { Microbes } \\
\text { co-cultured }\end{array}$ & $\begin{array}{c}\text { Deep } \\
\text { corneal } \\
\text { vessels } \\
(\mathrm{mm})\end{array}$ & $\begin{array}{l}\text { Treatment } \\
\text { (no. of } \\
\text { antibiotics) }\end{array}$ \\
\hline M & 2 & E. coli & 4 & 0 \\
\hline M & 2 & Haemophilus & 3 & $?$ \\
\hline $\mathrm{L}$ & 4 & S. epidermidis & 2 & 0 \\
\hline M (4ii) & 4 & S. pneumoniae & 0 & 2 \\
\hline $\mathrm{L}$ & 5 & S. epidermidis & 3 & 0 \\
\hline M (3ii) & 6 & Fungi, E. coli & 3 & 4 \\
\hline M & 6 & Pneumococci & 3 & 1 \\
\hline M & 9 & Serratia marcescens & 2 & 3 \\
\hline M & 9 & S. aureus & 3 & 1 \\
\hline $\mathrm{L}(5 \mathrm{i})$ & 12 & $\begin{array}{l}\text { S. pneumoniae, } \\
\text { S. epidermidis }\end{array}$ & 5 & 3 \\
\hline L (5ii) & 13 & S. epidermidis, $(\mathrm{G}-\mathrm{rod})$ & 5 & 4 \\
\hline
\end{tabular}

M, diagnosed 1-2 months after onset of first symptoms; L, diagnosed later than 2 months; (5i/5ii) etc. indicates the same eye on different occasions.

mixed infections, with a mean active treatment time of 66.25 weeks compared with a mean of 51 weeks for those with Acanthamoeba alone, although this did not reach statistical significance.

\section{Medical Management}

Propamidine and neomycin were most commonly used together (in 29 eyes). The combination of propamidine and PHMB was used alone in 13 eyes and all three drugs were used in another 12. Propamidine was used alone in 1 eye and a combination of between three and 12 drugs were used in the remaining 22 eyes including most commonly the above, paromomycin, itraconazole, clotrimazole, miconazole and ketoconazole. One patient also received acriflavine, polymyxin $\mathrm{B}$, colistin, crystal violet, methyl benzalkonium chloride, an experimental drug (R11/29) and brilliant green. Flurbiprofen was used in 42 of 72 cases, topical steroid in 49 of 77 eyes and systemic steroid was used in 4 of 72 cases, for the indications above (see Methods).

Chronically non-healing ulcers, potentially due either to uncontrolled infection or to cessation of the healing process, were reinvestigated with scrapes and cultures. In 3 eyes persistent Acanthamoeba was discovered and the anti-amoebal treatment was increased or changed. One also had a streptococcal superinfection, which was intensively treated. Of a further 10 eyes with non-healing ulcers, 7 had intense punctate corneal or conjunctival stain and pain, and drop toxicity was thought to be an aggravating factor; in 4 the ulcer was indolent with a necrotic surface. In 7 of these eyes propamidine and/or neomycin were reduced and given without preservatives or stopped; steroids were added in 6 eyes, botulinum toxin was used to give a therapeutic ptosis in 4 cases and debridement by lamellar keratectomy was performed in 2 .

Recurrent amoebal corneal disease occurred in 8 eyes. In 3 of these treatment had been reduced to a low level (four times daily propamidine, 6 times daily PHMB and twice daily propamidine plus PHMB respectively) when new small infiltrates or satellites appeared; all recovered quickly on increased anti-amoebal drop frequency. In 1 


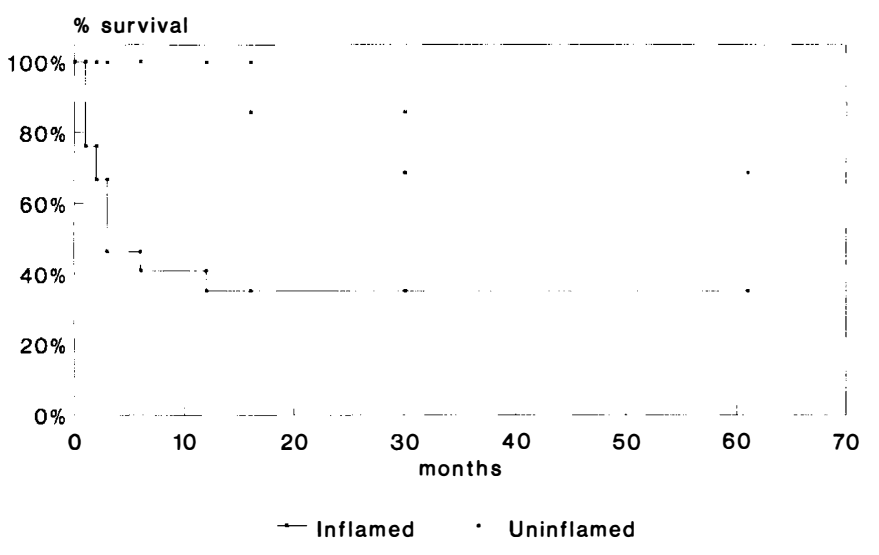

Fig. 5. Graft survival after Acanthamoeba keratitis.

complicated relapsing case a medical cure was achieved by adding PHMB, but the patient needed keratoplasty 3 months later when the eye was quiet. Four more severely affected eyes did not respond well to increased propamidine and neomycin (plus paromomycin in 1 and PHMB in 2) in spite of good in vitro sensitivities; 3 of them required 'hot' keratoplasty between 2 and 5 months later. The fourth developed glaucoma and cataract, and after cataract extraction first scleritis then finally phthisis ensued.

Patients received treatment as in-patients for a shorter time in 1992 than previously. Where hourly intensive initial therapy could not be given at home or there was severe sight-threatening disease, patients were admitted for treatment. The average total number of days as an inpatient for patients diagnosed and successfully treated in 1992 was 2.4 days $(n=20)$; this compares with 59 days required for those diagnosed in $1987(n=9)$, gradually reducing to 9 days in $1991(n=22)$.

\section{Surgical Therapy}

Keratoplasty was performed in 1 of 36 eyes (3\%) of the early-diagnosed cases, 6 of 17 eyes (35\%) of the intermediate group and 16 of 24 eyes $(66 \%)$ of the latepresenting groups. A total of 34 penetrating keratoplasties were done in 23 eyes.

Twenty-one procedures were performed in inflamed or 'hot' eyes (i.e. actively infected, perforated or with recurrence of infection in a graft) and 13 in uninflamed. Fig. 5 shows Kaplan-Meier survival curves for grafts in inflamed and uninflamed eyes, with probabilities of graft survival at 61 months being 0.35 and 0.69 respectively. The hazard ratio between the two was greatest at 12 months, at 7.34 (95\% confidence interval 2.36-22.9).

Two of 13 'cold' procedures failed: 1 at 16 months due to uncontrolled glaucoma in a non-compliant individual and 1 at 30 months due to secondary bacterial endophthalmitis. The mean follow-up time for survival grafts in uninflamed eyes was 31 months (range 3-80 months). Failures occurred after 13 of 21 procedures in inflamed eyes, from 2 weeks post-operatively to 6 months (mean 12 weeks). The mean follow-up time of surviving grafts in inflamed eyes was 40 months (range 2-88 months); 2 of these patients were lost to follow-up abroad at 2 and 6 months post-operatively respectively. The commonest cause of graft failure was recurrent Acanthamoeba infection (9 eyes), usually within days; glaucoma caused failure in 3 and phthisis in 1 . In these inflamed eyes, complications not resulting in failure included another Acanthamoeba recurrence and 3 more with glaucoma.

Keratoplasty was repeated once on 9 eyes. Four of these operations were successfully performed when the eye had become quiet, but of five 'hot' procedures, three failed. Two eyes underwent 'hot' second and third keratoplasties, utilising matched tissue in both cases for the latter, and only 1 of these survived.

\section{Outcome}

A medical cure was achieved in 63 of 73 eyes (86\%) (outcome data was only available in $73 / 77$ eyes, since 4 were lost to follow-up abroad) and 13 of 63 quiet eyes went on to 'cold' keratoplasty for visual reasons. In a further 4 eyes, after a first failed keratoplasty, a medical cure was effected before regrafting was performed.

The visual outcome is broken down by group in Fig. 6 . Overall, of the 73 eyes, 58 (79\%) have regained an acuity of between $6 / 4$ and $6 / 12$. A further 9 eyes (12\%) have useful vision of $6 / 18$ to $6 / 60$. One eye $(1 \%)$ has vision of counting fingers, $1(1 \%)$ has light perception, and $4(6 \%)$ have lost all perception of light.

The visual outcome was similar if the culture-positive cases are considered separately. Forty-six of the 73 eyes revealed Acanthamoeba on microscopy and/or culture of corneal specimens. Thirty-two of 46 (70\%) achieved final visual acuities of $6 / 4$ to $6 / 12,9(20 \%)$ achieved $6 / 18$ to $6 / 60,1(2 \%)$ counting fingers, $1(2 \%)$ light perception and $3(6 \%)$ were left without perception of light.

The reasons for a poor final outcome were multifactorial and interrelated in the 15 eyes with a final acuity of $6 / 18$ or less; they included late diagnosis (later than a month, $n=15)$, central scarring $(n=10)$, glaucoma $(n=5)$, resistant organisms $(n=2)$, microbial coinfection $(n=7)$, prolonged steroid before the diagnosis of Acanthamoeba was made ( $n=5)$, and failure of penetrating keratoplasty ( $n=7$ eyes, 11 keratoplasties). The principal cause of profound visual loss was uncontrolled glaucoma, whereas the commonest cause of a loss of acuity down to $6 / 24$ was axial corneal scarring.

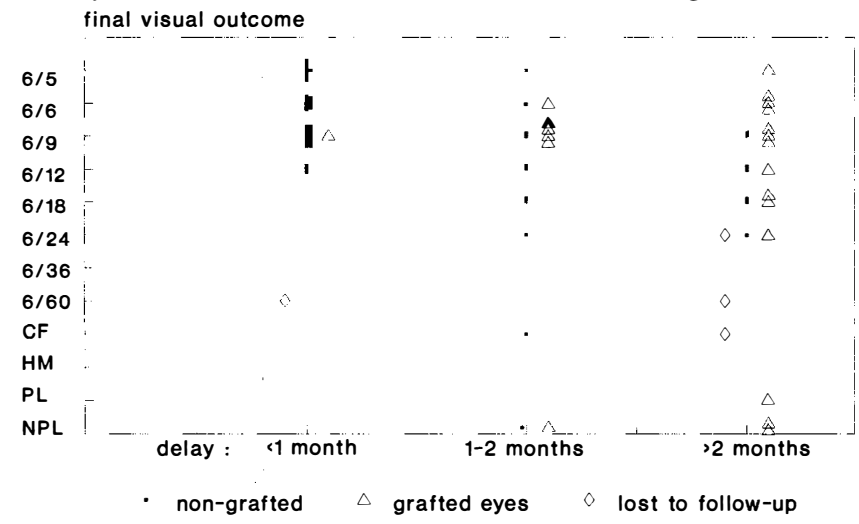

Fig. 6. Final visual outcome versus delay in presentation. 


\section{DISCUSSION}

We believe that the biggest single variable still affecting the progress and outcome of the disease is the length of time between first symptoms and the institution of the anti-amoebal regime. We have established that the presenting signs reflect generally deeper corneal involvement at presentation in later-presenting disease than in early disease. Clinical signs of severe disease including hypopyon, glaucoma and cataract are uniformly more common in late-presenting disease. A higher proportion of cases require penetrating keratoplasty and the final visual acuity is commensurately poorer. Long-standing atypical keratitides still come to light, and in the last 2 years we have seen tertiary referrals up to 15 months after onset, our series including several late referrals from abroad.

The laboratory staining, plating and culturing techniques required for identifying Acanthamoeba necessitate expertise in a dedicated microbiological department. Confirmation of the diagnosis is particularly important to distinguish acanthamoebal from bacterial or fungal keratitis, since the treatment is so different, and every effort should be made to obtain corneal material, contact lenses and their care systems for culture. Eight patients in this series had negative corneal scrapes but with both a typical clinical picture and culture-positive lenses or solutions we consider these presumed amoebal, although we are aware that $0-7 \%$ of non-affected wearers may have Acanthamoeba culture-positive lens cases. ${ }^{17}$ There is particularly strong justification for the inclusion of cases with perineural infiltrates since there is only one report of these occurring in any other context, in Pseudomonas keratitis ${ }^{18}$ (and this may have been polymicrobial).

The number of corneas with microbial co-infection (20/ 27) was striking, although not as high as the $58 \%$ reported by M. S. Osato (unpublished data, presented at the American Academy of Ophthalmology 1992). The presence of bacterial keratitis in combined infections, both clinically and on culture, may delay the diagnosis of Acanthamoeba keratitis and therefore appropriate treatment. The question of whether the bacteria are the primary infecting agent is still largely speculative. Acanthamoeba thrive on bacterial colonies in culture, particularly on Gram-negative rods, and may reflect combined infection of the lenses or cases. ${ }^{17,19,20}$ This sequence of events seems most likely where bacteria were isolated only days after the first symptoms were experienced. However, the corneal ulceration seen in later-presenting acanthamoebal infection is a recognised risk factor for bacterial superinfection. The majority of our cases support this latter mechanism for two reasons: the co-culture of fungus in 1 case and of more than one other bacterium in 8. Polymicrobial infections tend to be more virulent and difficult to treat and are significantly associated with the development of deep corneal neovascularisation (16/20 eyes with a mixed infection, vs. 8/57 with Acanthamoeba alone). Polymicrobial keratitis must be suspected if the features are atypical, there is a poor response to treatment or if new infiltrates appear. They should be investigated and treated aggressively, with intensive specific antibiotics.
Recurrence of active disease in the cornea was noted in 1 case each of the early and intermediate groups and in 6 of the late-diagnosed group. In 3 of these it appeared that patients had simply reduced their treatment too quickly, and more intensive therapy allowed control to be regained. In 1 case surgery followed slow but successful medical control, but in another 4 cases satisfactory medical control was not achieved. The reasons for the apparent occasional resistance of Acanthamoeba to combinations of agents that have shown excellent in vitro activity ${ }^{9.15}$ are not clear; there is recent evidence that the clinical response may not reflect laboratory sensitivities. ${ }^{21}$

The distinction between recurrent or persistent Acanthamoeba infection and non-infectious causes of failure to heal, such as drop toxicity or indolent ulcer, was critical since in the former the anti-amoebal treatment was intensified and augmented whereas in the latter it was usually reduced and steroids added. Propamidine ${ }^{22}$ and neomycin are both potentially toxic to the corneal epithelium; numerous patients reported stinging or pain on using one or other of the drops, which in turn were reduced or substituted with other anti-amoebal agents such as PHMB. Preservatives in the drops were also identified as likely toxic agents after intensive multiple drug therapy, and unpreserved agents were substituted. Steroids were increased in 6 patients in the hope of reducing the lytic process at the surface, but botulinum-toxin-induced ptosis $^{23}$ and debridement ${ }^{24.25}$ (by lamellar keratoplasty in our cases) were of most help in aiding re-epithelialisation in eyes with indolent ulcers.

Evaluation of the results of 34 keratoplasties is scarcely more encouraging than the earlier report by Ficker ${ }^{26}(16$ patients being common to the two series), who indicated a probability of graft survival of 0.2 in inflamed eyes at 60 months. We have shown a probability of survival of 0.35 at 61 months in inflamed eyes. However, the outcome of grafting uninflamed eyes was significantly better than inflamed, with a probability of survival at 61 months of 0.69 , failure occurring late in only 2 of 13 uninflamed cases, 1 who defaulted on her glaucoma treatment and the other who developed a stitch infection followed by endophthalmitis. Of 21 procedures in inflamed eyes 13 failed; 9 of these were due to rapidly recurring Acanthamoeba infection, 3 failed due to glaucoma and 1 became phthisical. Elective surgery on a quiet eye at a later stage when limbitis has subsided reduces the risk of intractable post-keratoplasty glaucoma. The success of medical treatment (63/73 having undergone medical cure, whether or not grafting followed) has meant that unless there is a fulminant corneal abscess, perforation or heavy infection of a recent graft, surgery in the presence of active Acanthamoeba is contraindicated until a medical cure has been effected.

Culture-negative eyes were generally those which had been diagnosed early and were more superficially involved, which may therefore have had a smaller infecting (and culturable) load. Other reasons for a negative culture may have included drying of the epithelial biopsy with rupture of trophozooites or infection by other organisms such as 
Valkampfid. ${ }^{27}$ Cultures were not pursued where medical treatment induced a marked improvement, so that early cases rarely had multiple or formal corneal biopsies, perhaps explaining their lower culture-positivity rate.

The final visual outcome was $6 / 12$ or better in 58 of 73 eyes (79\%) with presumed Acanthamoeba (data were available only for 73 of the 77 eyes originally diagnosed) and in 32 of $46(70 \%)$ of those that were tissue culturepositive. The outcome of those diagnosed before a month has been $6 / 12$ or better in every case (Fig. 6); nevertheless the prospects for those diagnosed later have also improved so that the majority $(21 / 33$, or $64 \%$ of those diagnosed after a month) also achieve $6 / 12$ or better. The 15 eyes with the poorest outcome were all diagnosed later than a month after first symptoms, and the commonest cause of profound visual loss was glaucoma.

The year 1992 may have been a milestone for Acanthamoeba keratitis in more than one way. Although more patients have presented to us almost every year since 1984, the number of non-lens-wearers, and wearers of nondisposable soft and rigid lenses has changed little. Our index of suspicion of Acanthamoeba is higher than ever before, and wearers of disposable lenses are now most painstakingly investigated. The commonest initial clinical diagnosis has changed from herpes simplex to Acanthamoeba keratitis and the mean interval between first symptoms and institution of treatment has shortened every year, to its current 9.3 days. The use of PHMB in combination with other anti-amoebal agents, recently reported to be effective and relatively non-toxic, ${ }^{15}$ has more consistently confined the period of intensive treatment to a matter of weeks. ${ }^{21}$ Finally, early diagnosis has largely made it possible to avoid surgery in inflamed eyes and persistence with medical therapy has allowed a primary medical cure in 63 of 73 eyes that were followed up. New patients presenting with Acanthamoeba keratitis may justifiably begin treatment with some optimism.

The authors would like to thank Mr. R. J. Buckley, Mr. A. D. McG. Steele and Mr. N. S. C. Rice for kindly allowing the inclusion of their patients in this study.

\section{REFERENCES}

1. Berger ST, Mondino BJ, Hoft RH, Donzis PB, Holland GN, Farley MK, Levenson JE. Successful medical management of Acanthamoeba keratitis. Am J Ophthalmol 1990;110: 395-403.

2. Moore MB, McCulley JP. Acanthamoeba keratitis associated with contact lenses: six consecutive cases of successful management. Br J Ophthalmol 1989;73:271-5.

3. Lindquist TD, Sher NA, Doughman DJ. Clinical signs and medical therapy of early Acanthamoeba keratitis. Arch Ophthalmol 1988;106:73-7.

4. Moore MB, McCulley JP, Kaufman HE, Robin JB. Radial keratoneuritis as a presenting sign in Acanthamoeba keratitis. Ophthalmology 1986;93:1310-5.

5. Tripathi RC, Monninger RHG, Tripathi BJ. Contact-lens associated Acanthamoeba keratitis: a report from the USA. Fortschr Ophthalmol 1989;86:67-71.

6. Bacon AS, Dart JKG, Ficker LA, Matheson M, Wright P.
Acanthamoeba keratitis: the value of early diagnosis. Ophthalmology 1993;100:1238-43.

7. John T, Lin J, Sahm D, Rockey JH. Effects of corticosteroids in experimental Acanthamoeba keratitis. Rev Infect Dis 1991;13s:s440-2.

8. Ficker L, Seal D, Warhurst D, Wright P. Acanthamoeba keratitis: resistance to medical therapy. Eye 1990;4:835-8.

9. Osato MS, Robinson NM, Wilhelmus KR, Jones DB. In vitro evaluation of antimicrobial compounds for cysticidal activity against Acanthamoeba. Rev Infect Dis 1991;13s: s431-5.

10. Cohen EJ, Parlato CJ, Arentsen JJ, Genvert GI, Eagle RC, Wieland MR, Laibson PR. Medical and surgical treatment of Acanthamoeba keratitis. Am J Ophthalmol 1987;103: 615-25.

11. McLellan K, Coster DJ. Acanthamoebic keratitis diagnosed by paracentesis and biopsy and treated with propamidine. $\mathrm{Br}$ J Ophthalmol 1989;71:734-6.

12. Ficker LA. Acanthamoeba keratitis: the quest for a best prognosis. Eye 1988;2 (Suppl):S37-45.

13. Silvany RE, Luckenbach MW, Moore MB. Paraffin-embedded sections of corneal tissue with calcofluor white. Arch Ophthalmol 1987;105:1336-7.

14. Robin JB, Chan R, Rao NA, Sharma S, Srinivasan M. Fluorescein-conjungated lectin visualisation of fungi and Acanthamoeba in infectious keratitis. Ophthalmology 1989; 96:1198-1202.

15. Larkin DFP, Kilvington S, Dart JKG. Treatment of Acanthamoeba keratitis with polyhexamethylene biguanide. Ophthalmology 1992;99:185-91.

16. Stapleton F, Dart JKG, Minassian D. Nonulcerative complications of contact lens wear: relative risks for different lens types. Arch Ophthalmol 1992;110:1601-6.

17. Devonshire P, Munro FA, Abernethy C, Clark BJ. Microbial contamination of contact lens cases in the west of Scotland. Br J Ophthalmol 1993;77:41-5.

18. Feist R, Sugar J, Tesler H. Radial keratoneuritis in Pseudomonas keratitis. Arch Ophthalmol 1991;109:774-5.

19. Larkin DF, Kilvington S, Easty DL. Contamination of contact lens storage cases by Acanthamoeba and bacteria. Br J Ophthalmol 1990;74:133-5.

20. Larkin DFP, Easty DL. External eye flora as a nutrient source for Acanthamoeba. Graefes Arch Clin Exp Ophthalmol 1990;228:458-60.

21. Elder MJ, Kilvington S, Dart JKG. A clinicopathological study of in vitro sensitivity testing and Acanthamoeba keratitis. Invest Ophthalmol Vis Sci (in press).

22. Johns KJ, Head WS, O'Day DM. Corneal toxicity of propamidine. Arch Ophthalmol 1988;106:68-9.

23. Kirkness C, Adams GGW, Dilly PN, Lee JP. Botulinum toxin A-induced protective ptosis in corneal disease. Ophthalmology 1988;95:473-80.

24. Holland GN, Donzis PB. Rapid resolution of early Acanthamoeba keratitis after epithelial debridement (letter). Am J Ophthalmol 1987;104:87-9.

25. Ishibashi Y, Matsumoto Y, Kabata T, Watanabe R, Hommura S, Yasuraoka K, Ishii K. Oral itraconazole and topical miconazole for Acanthamoeba keratitis. Am J Ophthalmol 1990;109:121-6.

26. Ficker LA, Kirkness C, Wright P. Prognosis for keratoplasty in Acanthamoeba keratitis. Ophthalmology 1993;100: 105-10.

27. Kirkness C, Aitken D, Gavin M, Hay J, Lee W, Seal D. Valkampfid keratitis simulating Acanthamoeba infection associated with disposable contact lens wear (an overlooked diagnosis?). Invest Ophthalmol Vis Sci 1993;34(ARVO Suppl):853. 